Pacific Journal of Mathematic 


\title{
SET FUNCTIONS ASSOCIATED WITH LEBESGUE AREA
}

\author{
EDward Silverman
}

1. Introduction. It will be convenient, for the purposes of this paper, to define Lebesgue area as in [6]. This definition is applicable to surfaces in metric spaces and is equivalent to the usual definition for surfaces in Euclidean space.

There is described in [7] a method for generating from a continuous function defined on a square into a metric space, a monotone function with range in $\mathrm{m}$, the space of bounded sequences [1], having the same Lebesgue area. A corresponding remark holds for Fréchet surfaces instead of continuous functions.

Suppose that $\varnothing$ is a surface and that is obtained from $\&$ by the procedure referred to. The purpose of this paper is to show the existence of set functions whose values on elementary configurations of $m$ agree with the elementary areas of the configurations, and whose values on the point set occupied by are equal to the Lebesgue area of $\$$. We shall give definitions for two such set functions; for one of these functions it is necessary to assume that the Lebesgue area of $\&$ is finite in order to be sure that the equality holds.

The set functions can be interpreted for subsets of $E_{n}$. If a surface in $E_{n}$ admits a monotone representation, then the value of each of the set functions on the point set carrying the surface is, with the proviso mentioned above, equal to the Lebesgue area of the surface.

2. Preliminary remarks. The definition of Lebesgue area which we shall use is given in this section. We shall see that there is no loss in generality in supposing that all of the surfaces with which we shall be concerned are in $m$.

We list here some definitions and notations that will be used in the sequel. If $D$ is a domain in the plane and if a subset $\mathscr{V}$ of some topological space is homeomorphic to $D$, then $D$ is a 2 -domain. If $E$ is a set, then $\bar{E}, E^{0}$, and $E^{*}$ will denote its closure, interior, and boundary, respectively. We reserve the letters $f$ and $g$ to represent linear functionals on $m$ of norm one. For fixed $f$ and $g, \pi f g$ is the transformation from $m$ to $E_{2}$ defined by $\pi^{f g}(a)=(f(a), g(a))$ for each $a \in m$. If $a, b$, and $c$ are the vertices of a triangle in $m$, then the area of the triangle is, by definition,

Received March 23, 1951. This paper was written with the financial support of the Research Corporation.

Pacific J. Math. 2 (1952), 243-250 


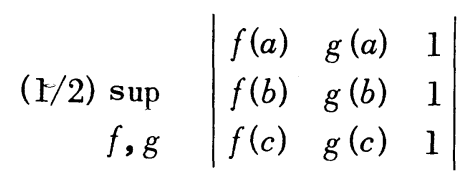

where, as indicated above, $f$ and $g$ are linear functionals of norm one.

If $\varnothing$ is a surface, then $L(\&)$ and $P(\&)$ are the Lebesgue and Peano areas of \$. Similarly, $L(x)$ and $P(x)$ are the corresponding areas of $x$, where $x$ is a suitable continuous function. If $Q$ is a 2-domain in $m, \phi$ is a transformation from $\bar{D}$ to $E_{2}$, and $z \in E_{2}$, then $M(\phi, z)$ is the value of Federer's multiplicity function $M$ determined by $\phi$ at $z$ [4].

We denote the unit square by $Q$ and the surface of the unit sphere by $\Sigma$. Let $\psi$ be a transformation from $Q$ onto $\Sigma$ which is topological on $Q^{0}$ and constant on $Q^{*}$. If $x$ is continuous on $Q$ and constant on $Q^{*}$, then $\bar{x}$ is defined on $\Sigma$ by $\bar{x}(\psi(q))=x(q)$. In general, the composition of two functions $F$ and $G$ is denoted by $F * G$. Hence we can write $x=\bar{x} * \psi$. If $D$ is contained in the domain of a function $F$, then $F \mid D$ is the function $F$ restricted to $D$.

We recall here the definitions of Peano and Lebesgue areas [6].

Let $x$ be continuous on $Q$ into $m$.

If $D$ is a domain contained in $Q^{\circ}$, and

$$
G(x, D)=\sup _{f, g} \iint_{E_{2}} M\left(\pi^{f g} * x \mid \bar{D}, z\right) d z,
$$

then

$$
P(x)=\sup _{\sigma} \sum_{D \in \sigma} G(x, D),
$$

where $\sigma$ is a finite disjoint family of domains contained in $Q^{0}$.

We define the Peano area of a function defined on $\Sigma$ in an analogous inanner. For convenience we suppose that $\sigma$ does not consist of $\Sigma$ alone.

In [6], Lebesgue area was defined by means of quasilinear functions. It can be shown, as in [5], that this definition is equivalent to that given below.

A polyhedron is a surface which admits a representation $x$ on $Q$ such that there exists a curvilinear triangulation of $Q$ consisting of curvilinear triangles $t_{1}, \cdots, t_{n}$, and for each $i(i=1, \cdots, n)$,

(i) $x \mid t_{i}$ is topological,

(ii) $x\left(t_{i}\right)$ is a nondegenerate triangle (in $m$ ), the vertices of $x\left(t_{i}\right)$ being the images of the vertices of $t_{i}$. 
If $P$ is a polyhedron in $m$, then it can be shown, just as the corresponding statement is proved for polyhedra in $E_{n}$, that $P(P)$ is equal to the elementary area of $P$.

If $\delta$ is a surface (in $m$ ), then

$$
L(\&)=\lim _{D(\mathcal{D}, \infty) \rightarrow 0} \inf _{0}\{\text { elementary area of } P\},
$$

where $D(P, \&)$ is the Fréchet distance between $P$ and \&.

The Lebesgue area of a surface represented on $\Sigma$ is defined in an analogous manner.

If $\delta^{\prime \prime}$ is a surface in a metric space $m$, then there exists a surface $\&^{\prime}$ in $m$ which is isometric to $\varnothing^{\prime \prime}$ (if $x^{\prime \prime}$ is a representation of $\varnothing^{\prime \prime}$, then there exists a representation $x^{\prime}$ of $\delta^{\prime}$ such that $\left\|x^{\prime}(p)-x^{\prime}(q)\right\|=d\left(x^{\prime \prime}(p), x^{\prime \prime}(q)\right)$, the distances being in $m$ and $m$ respectively). We define $L\left(\delta^{\prime \prime}\right)$ to be $L\left(\delta^{\prime}\right)$. The definition is valid since Lebesgue area in $m$ satisfies Kolmogoroff's principle. As noted earlier, it is this definition of Lebesgue area which we shall use.

3. The set function $\mu$. We make the following definitions.

DEFINITION 3.1. Let $S^{\prime} \subset m$, and let $\sigma^{\prime}$ be a finite family of disjoint 2-domains contained in $S^{\prime}$. Then

$$
\mu\left(S^{\prime}\right)=\sup _{\sigma^{\prime}} \sum_{D \in \sigma^{\prime}} \mu^{\prime}(D)
$$

where

$$
\mu^{\prime}(\mathcal{D})=\sup _{f, g} \iint_{E_{2}} M\left(\pi^{f g} \mid \overline{\mathcal{D}}, z\right) d z .
$$

(If $\sigma^{\prime}$ is empty, then $\sum_{D} \mathcal{E}_{\sigma} \mu^{\prime}(\mathbb{D})=0$. )

DEFINITION 3.2. A compact subset of $m$ consisting of a denumerable number of points, line-segments, triangles, and so on, is an elementary configuration in $m$. If $E$ is an elementary configuration, then $|E|$ is the elementary area of $E$.

We observe that if $S^{\prime}$ is the monotone image of a 2-cell, then $\mu$ is additive with respect to its cyclic elements. If $E$ is an elementary configuration, then $\mu(E)=|E|$.

Now let $x$ be continuous on $Q$ into $m$. Define $\tilde{x}$ on $Q \times Q$ by

$$
\tilde{x}(p, q)=\inf \operatorname{diam} x(\zeta([0,1]))
$$


for all continuous functions $\zeta$ defined on $[0,1]$ such that $\zeta(0)=p, \zeta(1)=q$, and range $\zeta=\zeta([0,1])$ is contained in $Q$. If $\left\{p_{i}\right\}$ is an everywhere dense sequence of points in $Q$, define $x^{\prime}$ on $Q$ into $m$ by $x^{\prime}(q)=\left\{\tilde{x}\left(p_{i}, q\right)\right\}$. Then $x^{\prime}$ is monotone and $L\left(x^{\prime}\right)=L(x)[7]$.

It is necessary, for our purposes, to extend this result to the case where $x$ is defined on $\Sigma$. If $p \in \Sigma, q \in \Sigma$, then $\zeta$ is admissible if range $\zeta \subset \Sigma$. Then $\tilde{x}$ and $x^{\prime}$ are defined as before. In order to show that $L(x)=L\left(x^{\prime}\right)$, let $y$ be defined on $Q$ by $y=x * \psi$. The reader can verify that $x=\bar{y}$ and $(\bar{y})^{\prime}=\bar{y}^{\prime}$. We assume the results of Lemma 3.2 to obtain $L(\bar{y})=L(y)$ and $L\left(\bar{y}^{\prime}\right)=L\left(y^{\prime}\right)$. Thus we have $L(x)=L(\bar{y})=L(y)=L\left(y^{\prime}\right)=L\left(\bar{y}^{\prime}\right)=L\left(x^{\prime}\right)$.

LEMMA 3.1. If $x$ is light on $Q$ (or $\Sigma$ ), and $D$ is a domain in $Q^{0}$ (a 2-domain in $\Sigma)$, then $M\left(\pi^{f g} *(x \mid D), z\right)=M\left(\pi^{f g} \mid x^{\prime}(D), z\right)$.

The proof is almost evident since $x^{\prime}$, being monotone as well as light, is a homeomorphism.

LEMMA 3.2. If $x$ is continuous on $Q$ and constant on $Q^{*}$, then $P(\bar{x})=P(x)$ and $L(\bar{x})=L(x)$.

The argument of Cesari in [3] is valid here.

We are now in a position to compare $\mu\left(x^{\prime}(Q)\right.$ with $P\left(x^{\prime}\right)$.

LEMmA 3.3. If $x$ is light on $Q$ or light on $Q^{0}$ and constant on $Q^{*}$, then $\mu\left(x^{\prime}(Q)\right)=P\left(x^{\prime}\right)$.

The result follows from Lemma 3.1 or Lemmas 3.1 and 3.2.

LEMMA 3.4. If $x$ is continuous on $Q$ into $m$, then $P\left(x^{\prime}\right)=\mu\left(x^{\prime}(Q)\right)$.

Proof. The equality is a result of the cyclic additivity of $P$ and $\mu$, the preceding lemma, and the following three statements [5]:

(i) If $x^{\prime}(Q)$ is a 2-cell, then there exists a light function $y^{\prime}$ which is Fréchet equivalent to $x^{\prime}$.

(ii) If $x^{\prime}(Q)$ is a 2-sphere, then there exists a function $y^{\prime}$ which is Fréchet equivalent to $x^{\prime}$ and which is light in $Q^{0}$ and constant on $Q^{*}$.

(iii) If $D\left(x^{\prime}, y^{\prime}\right)=0$, then $x^{\prime}(Q)=y^{\prime}(Q)$.

LEMMA 3.5. If $P(x)=L(x)$ and $P\left(x^{\prime}\right) \geqq P(x)$, then $L(x)=\mu\left(x^{\prime}(Q)\right)$.

Proof. We have $P\left(x^{\prime}\right) \leqq L\left(x^{\prime}\right)=L(x)=P(x) \leqq P\left(x^{\prime}\right)$, and so $L(x)=$ $P\left(x^{\prime}\right)$. The result now follows from the preceding lemma. 
Suppose that we use, instead of $x^{\prime}$, the function $x^{\prime \prime}$ defined by $x^{\prime \prime}(q)=$ $\left\{z^{k}(q)\right\}$ for $q \in Q$, where

$$
\begin{aligned}
& z^{k}(q)=x^{\prime(k+1) / 2}(q) \\
& z^{k}(q)=x^{k / 2}(q)
\end{aligned}
$$

if $k$ is odd,

if $k$ is even .

It is evident that $\left\|x^{\prime \prime}(p)-x^{\prime \prime}(q)\right\|=\left\|x^{\prime}(p)-x^{\prime}(q)\right\|$ for $p, q \in Q$, and so $L\left(x^{\prime \prime}\right)=L\left(x^{\prime}\right)=L(x)$. It is not hard to see that $P\left(x^{\prime}\right) \geqq P(x)$. We can now summarize our results.

TheOREM 3.1. If $L(x)<+\infty$, then $L(x)=\mu\left(x^{\prime}(Q)\right)$. If $P(x)=L(x)$,

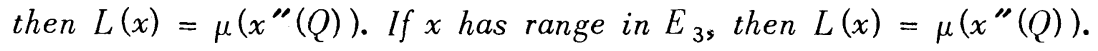

The last statement of the theorem results when we observe that we may consider $E_{3}$ as a subspace of $m$, and then use the fact that $P(x)=L(x)$ [2].

4. The set function $\lambda$. In defining $\lambda$ we leaned heavily on Peano area. We shall define a (possibly) new set function $\lambda$ by relying upon Lebesgue area.

Let $\mathcal{E}$ be the family of elementary configurations of $m$, and let $\mathcal{F}$ be the family of subsets of $m$ each homeomorphic to an element of $\varepsilon$.

In each class of homeomorphic subsets of $m$ we introduce a metric $d$ defined by

$$
d(A, B)=\inf _{h} \sup _{p}\|p-h(p)\|,
$$

where $h$ is a homeomorphism of $A$ onto $B$.

Lemma 4.1. For each $F \in \mathcal{F}$ there exists a sequence $\left\{E_{n}\right\}, E_{n} \in \mathcal{E}$, such that $d\left(E_{n}, F\right) \rightarrow 0$.

Proof. There exists a set $E \in \mathcal{E}$ and a homeomorphism $h$ such that $F=h(E)$. Since $E$ is compact, $h$ is uniformly continuous and so, for $\epsilon>0$, there exists a $\delta>0$ such that $\|h(p)-h(q)\|<\epsilon$ if $\|p-q\|<\delta$. Let $T_{n}$ be a triangulation of $E$ of mesh less than $\delta$. Define $h_{n}$ on $E$ by putting $h_{n}(p)=h(p)$ if $p$ is a vertex of $T_{n}$ and extending $h_{n}$ to be linear on each $k$-simplex of $T_{n}$. Then if $q \in E$, and $p$ is a vertex of a $k$-simplex of $T_{n}$ containing $q$, we have

$$
\begin{aligned}
\left\|h(q)-h_{n}(q)\right\| \leqq & \|h(q)-h(p)\|+\left\|h(p)-h_{n}(p)\right\|+ \\
& \left\|h_{n}(p)-h_{n}(q)\right\|<\epsilon+0+\epsilon=2 \epsilon .
\end{aligned}
$$

We now let $E_{n}=h_{n}(E)$. 
DEFINITION 4.1. Define $\lambda^{\prime}$ on $\mathcal{F}$ by

$$
\begin{gathered}
\lambda^{\prime}(F)=\lim _{\substack{d(E, F) \rightarrow 0 \\
E \in \mathcal{E}}}|E|, \\
\\
E \in
\end{gathered}
$$

and $\lambda$ on subsets of $m$ by

$$
\lambda(S)=\sup _{\sigma F \in} \sum_{\sigma} \lambda^{\prime}(F),
$$

where $\sigma$ is a finite family of disjoint subsets of $S$ and $\sigma \in \mathcal{F}$.

We note the following result.

LEMMA 4.2. If $d\left(A_{n}, A\right) \rightarrow 0$, then $\lambda(A) \leqq \lim _{n \rightarrow \infty} \inf \lambda\left(A_{n}\right)$.

We require the following information from [6]. If $\pi$ is a plane (in $m$ ), then there exists a projection $\pi^{*}$ of $m$ onto $\pi$ such that $\left|\pi^{*}(\Delta)\right| \leqq|\Delta|$ for each triangle $\Delta$. Furthermore, if $p$ and $q$ are points of $m$ then $\left\|\pi^{*}(p)-\pi^{*}(q)\right\| \leqq$ $2\|p-q\|$.

Lemma 4.3. If $E \in \mathcal{E}$, and $\sigma$ is a finite family of disjoint subsets $F$ of $E$, each $F \in \mathcal{F}$, then $\sum_{F \in \sigma} \lambda(F) \leqq|E|$.

The proof will be sketched for the special case where $E$ consists of a single triangle $T$ in a plane $\pi$. Let $F \in \sigma$. By virtue of the remarks preceding this lemma, we may assume, without loss of generality, that there exists a sequence $\left\{E_{n}\right\}, E_{n} \in \mathcal{E}$, with $E_{n} \subset \pi, d\left(E_{n}, F\right) \rightarrow 0$, and $\left|E_{n}\right| \rightarrow \lambda^{\prime}(F)$. If $\tilde{E} \in \mathcal{E}$, and $\tilde{E} \subset F^{0}$, then $\tilde{E} \subset E_{n}$ for sufficiently large $n$. Hence $\lambda^{\prime}(F) \geqq$ Lebesgue measure of $F^{0}[6]$.

Let $\sigma^{\prime} \subset \mathcal{E}$ be a family of disjoint subsets of $F$. Then it is easy to see that $\sum_{F^{\prime}} \in \sigma_{\sigma^{\prime}} \lambda^{\prime}\left(F^{\prime}\right) \leqq$ Lebesgue measure of $F^{0}$, and so $\lambda(F)=$ Lebesgue measure of $F^{0}$. The lemma results for the special case considered.

The general case follows when the lemma has been proved under the assumption that $E$ consists of a finite number of triangles.

Lemma 4.4. If $F \in \mathcal{F}$, then $\lambda(F)=\lambda^{\prime}(F)$.

Proof. It is sufficient to show that $\lambda(F) \leqq \lambda^{\prime}(F)$. Let $\sigma$ be as in Definition 4.l. If $d\left(E_{n}, F\right) \rightarrow 0$ such that $\left|E_{n}\right| \rightarrow \lambda^{\prime}(F)$, then there exist homeomorphisms $h_{n}$ of $F$ onto $E_{n}$ with $d\left(F_{n}^{k}, F^{k}\right) \rightarrow 0$, where $F^{k} \in \sigma$ and $F_{n}^{k}=h_{n}\left(F^{k}\right)$. We use the preceding lemma and the lower semi-continuity of $\lambda$ to obtain $\sum_{F^{k} \in \sigma} \lambda\left(F_{n}^{k}\right) \leqq\left|E_{n}\right|$ and $\lambda\left(F^{k}\right) \leqq \lim _{\inf _{n \rightarrow \infty}} \lambda\left(F_{n}^{k}\right)$. Consequently 


$$
\begin{aligned}
& \sum_{F^{k} \in} \lambda\left(F^{k}\right) \leqq \sum_{F^{k}} \sum_{\sigma}\left\{\lim _{n \rightarrow \infty} \inf \lambda\left(F_{n}^{k}\right)\right\} \\
& \leqq \lim _{n \rightarrow \infty} \inf \sum_{F^{k}} \in_{\sigma} \lambda\left(F_{n}^{k}\right) \leqq \lim _{n \rightarrow \infty} \inf \left|E_{n}\right|=\lambda^{\prime}(F),
\end{aligned}
$$

and the lemma follows.

LEMMA 4.5. If $x$ is light on $Q$, then $L(x)=\lambda\left(x^{\prime}(Q)\right)$.

Proof. If $\phi^{\prime}$ is the (Fréchet) surface determined by $x^{\prime}$, then there exists a sequence of polyhedra $P_{n}$ such that $D\left(P_{n}, \varnothing^{\prime}\right) \rightarrow 0$ and \{elementary area of $P_{n}$ \} $\rightarrow L\left(\delta^{\prime}\right)=L\left(x^{\prime}\right)$. We may suppose that $P_{n}$ has a topological representation $\pi_{n}$ on $Q$. Since $\pi_{n}(Q) \in \mathcal{E}$ for each $n$, and $d\left(\pi_{n}(Q), x^{\prime}(Q)\right)=D\left(P_{n}\right.$, $\left.\phi^{\prime}\right) \rightarrow 0$, we see that

$$
\lambda\left(x^{\prime}(Q)\right)=\lambda^{\prime}\left(x^{\prime}(Q)\right) \leqq \lim _{n \rightarrow \infty} \inf _{n}\left|\pi_{n}(Q)\right|=L\left(x^{\prime}\right)=L(x)
$$

Now if $E_{n} \rightarrow x^{\prime}(Q)$ so that $\left|E_{n}\right| \rightarrow \lambda^{\prime}\left(x^{\prime}(Q)\right)$, then we define $x_{n}^{\prime}$ on $Q$ by $x_{n}^{\prime}=h_{n} * x^{\prime}$, where $h_{n}$ is a homeomorphism of $x^{\prime}(Q)$ onto $E_{n}$ such that $\sup _{p} \epsilon_{x^{\prime}(Q)}\left\|p-h_{n}(p)\right\|<d\left(x^{\prime}(Q), E_{n}\right)+1 / n$. It is clear that $x_{n}^{\prime}$ represents a polyhedron $D_{n}^{\prime}$ whose elementary area is $\left|E_{n}\right|$. Also, $D\left(x_{n}^{\prime}, x^{\prime}\right)<d\left(E_{n}, x^{\prime}(Q)\right)$ $+1 / n$. Therefore $D\left(x_{n}^{\prime}, x^{\prime}\right) \rightarrow 0$ and

$$
L\left(x^{\prime}\right) \leqq \lim _{n \rightarrow \infty} \inf L\left(x^{\prime}\right)=\lim _{n \rightarrow \infty} \inf \left|E_{n}\right|=\lambda^{\prime}\left(x^{\prime}(Q)\right) \leqq \lambda\left(x^{\prime}(Q)\right) \text {. }
$$

LEMMA 4.6. If $x$ is light in $Q^{0}$ and constant on $Q^{*}$, then $L(x)=\lambda\left(x^{\prime}(Q)\right)$.

The proof that $L(\bar{x})=\lambda\left(\bar{x}^{\prime}(\Sigma)\right)$ is similar to that of Lemma 4.5. The lemma results from the observation that $L(x)=L(\bar{x})=\lambda\left(\bar{x}^{\prime}(\Sigma)\right)=\lambda\left(x^{\prime}(Q)\right)$.

THEOREM 4.1. We have $L(x)=\lambda\left(x^{\prime}(Q)\right)$.

The proof of this theorem is similar to that of Lemma 3.4.

\section{REFERENCES}

1. S. Banach, Théorie des opérations lineaires, Monografje Matematyczne 1 Warsaw, 1932.

2. L. Cesari, Caratterizzazione analitica delle superficie continue di area finita secondo Lebesgue, Ann. Scuola Norm. Super. Pisa (2), 10 (1941), 253-294; 11 (1942), $1-42$.

3. , Sulle superficie di Fréchet, Rivista Mat. Univ. Parma, 1 (1950), 19-44. 
4. H. Federer, Essential multiplicity and Lebesgue area, Proc. Nat. Acad. Sci. U.S.A. 34 (1948), 611-616.

5. T. Rado, Length and area, Colloquium Publications Amer. Math. Soc., 30, New York 1948.

6. E. Silverman, Definitions of Lebesgue area for surfaces in metric spaces, Rivista Mat. Univ. Parma, 2 (1951), 47-76.

7. , An intrinsic property of Lebesgue area, Rivista Mat. Univ. Parma, 2 (1951), 195-201.

Kenyon College 


\section{PACIFIC JOURNAL OF MATHEMATICS}

\section{EDITORS}

R. M. RoBINSON

University of California Berkeley 4, California

\section{*R. P. Dilworth}

Califomia Institute of Technology Pasadena 4, California

\section{E. F. BECKENBACH, Managing Editor}

University of California

Los Angeles 24, California

*During the absence of Herbert Busemann in 1952.

\section{ASSOCIATE EDITORS}

\author{
R. P. DILWORTH \\ HERBERT FEDERER \\ MARSHALL HALL
}

\author{
P. R. HALMOS \\ HEINZ HOPF
}

R. D. JAMES

\author{
BØRGE JESSEN \\ PAUL LÉVY \\ GEORGE PÓLYA
}

\author{
J. J. STOKER \\ E. G. STRAUS
}

KÖSAKU YOSIDA

\section{SPONSORS}

UNIVERSITY OF BRITISH COLUMBIA

CALIFORNIA INSTITUTE OF TECHNOLOGY

UNIVERSITY OF CALIFORNIA, BERKELEY

UNIVERSITY OF CALIFORNIA, DAVIS

UNIVERSITY OF CALIFORNIA, LOS ANGELES

UNIVERSITY OF CALIFORNIA, SANTA BARBARA

OREGON STATE COLLEGE

UNIVERSITY OF OREGON

\author{
UNIVERSITY OF SOUTHERN CALIFORNIA \\ STANFORD UNIVERSITY \\ WASHINGTON STATE COLLEGE \\ UNIVERSITY OF WASHINGTON \\ AMERICAN MATHEMATICAL SOCIETY \\ NATIONAL BUREAU OF STANDARDS, \\ INSTITUTE FOR NUMERICAL ANALYSIS
}

Mathematical papers intended for publication in the Pacific Journal of Miathematics should be typewritten (double spaced), and the author should keep a complete copy. Manuscripts may be sent to any of the editors. All other communications to the editors should be addressed to the managing editor, E. F. Beckenbach, at the address given above.

Authors are entitled to receive 100 free reprints of their published papers and may obtain additional copies at cost.

The Pacific Journal of Mathematics is published quarterly, in March, June, September, and December, by the University of California, Berkeley 4, California. The price per volume (4 numbers) is $\$ 8.00$; single issues, $\$ 2.50$. Special price to individual faculty members of supporting institutions and to individual members of the American Mathematical Society: $\$ 4.00$ per volume; single issues, $\$ 1.25$.

Subscriptions, orders for back numbers, and changes of address should be sent to the publishers, University of California Press, Berkeley 4, California.

Printed at Ann Arbor, Michigan. Entered as second class matter at the Post Office, Berkeley, California.

\section{UNIVERSITY OF CALIFORNIA PRESS • BERKELEY AND LOS ANGELES}




\section{Pacific Journal of Mathematics}

\section{Vol. 2, No. $2 \quad$ February, 1952}

L. Carlitz, Some theorems on Bernoulli numbers of higher order...

Watson Bryan Fulks, On the boundary values of solutions of the heat equation........................................ 141

John W. Green, On the level surfaces of potentials of masses with fixed center of gravity................................... 147

Isidore Heller, Contributions to the theory of divergent series .......... 153

Melvin Henriksen, On the ideal structure of the ring of entire functions . . . 179

James Richard Jackson, Some theorems concerning absolute neighborhood retracts........................................ 185

Everett H. Larguier, Homology bases with applications to local connectedness ................................... 191

Janet McDonald, Davis's canonical pencils of lines ................ 209

J. D. Niblett, Some hypergeometric identities . . . . . . . . . . . . . . . . . . 219

Elmer Edwin Osborne, On matrices having the same characteristic equation...................................... 227

Robert Steinberg and Raymond Moos Redheffer, Analytic proof of the

Lindemann theorem ................................. 231

Edward Silverman, Set functions associated with Lebesgue area ......... 243

James G. Wendel, Left centralizers and isomorphisms of group algebras . . 251

Kosaku Yosida, On Brownian motion in a homogeneous Riemannian space. 